\title{
Multivisceral resection of locally advanced colorectal cancer in an African referral centre
}

\author{
OA Oke, $(\mathbb{D}$ EDT Coetzee, $(\mathbb{D}$ C Warden, $(\mathbb{D}$ PA Goldberg, $(\mathbb{D}$ A Boutall $\mathbb{D}$ \\ Colorectal Surgery, Department of General Surgery, University of Cape Town, South Africa
}

Corresponding author, email: emile@rectalsurgery.co.za

Background: Colorectal cancer (CRC) is common and often presents with advanced disease in Africa. Multivisceral resection (MVR) improves survival in locally advanced (T4b) CRC. The aim was to describe the management and outcomes of patients with clinical T4b CRC without metastatic disease who underwent MVR.

Methods: A retrospective review of patients with T4 CRC who underwent MVR between January 2008 and December 2013.

Results: Four hundred and ninety-four patients were included. Of the 158 with suspected T4 cancer, 44 had MVR, of which one was excluded due to metastases. The mean age was 64 years. The male to female ratio was 1:1. The most commonly resected extra-colorectal structure was the abdominal wall (21\%). The median survival was 68 months (SD 13.9). The 5-year disease free (DFS) and overall survival (OS) were $46 \%$ and $55 \%$, respectively. Survival of patients with colon and rectum cancer was similar. Intraoperative tumour spillage, vascular/perineural invasion, and anastomotic leakage were independent predictors of survival.

Conclusion: Multivisceral resection of locally advanced (T4b) CRC is feasible in the African context. Complete resection improves survival and should be the goal.

Keywords: colorectal cancer, multivisceral resection, locally advanced

\section{Introduction}

Colorectal cancer (CRC) is the third most common cancer worldwide, and the third most common cause of cancer death. ${ }^{1-3}$ The incidence is higher in the developed world, but mortality is disproportionately high in Africa. ${ }^{3}$ Delayed presentation, absence of screening programs, and inadequate diagnostic and treatment facilities have been suggested as possible contributing factors. ${ }^{3}$ In a West-African study, the majority of cases (99\%) were advanced at the time of presentation. Stage 4 disease comprised $58 \%$ of presenting cases and Stage 3 comprised $41 \%$. Only $1 \%$ of cases presented with early disease. ${ }^{4}$

Multivisceral resection (MVR) of locally advanced colorectal cancer may lead to 5-year overall survival rates between $49-76.6 \% .^{5-11}$ When complete resection is not achieved, median survival of 11.6 months has previously been reported. ${ }^{12}$ MVR involves the resection of primary cancer coupled with an en bloc resection of part, or the entire adjacent viscera involved through contiguous spread of disease. It may be technically demanding and may be associated with increased morbidity and mortality. ${ }^{7}$

With the high incidence of advanced disease at presentation of $\mathrm{CRC}$ in sub-Saharan Africa, adequate training in multivisceral resection techniques may reduce the morbidity and mortality of this disease. We describe the experience of a tertiary referral centre in Cape Town, South Africa.

\section{Materials and methods}

The hospital folders and surgical operations database of the Colorectal Surgery Unit of Groote Schuur Hospital,
University of Cape Town, South Africa were retrospectively reviewed.

We analysed all patients with clinically suspected T4b colon and rectal cancers, who had MVR of their tumour with curative intent. Clinical suspicion was defined as preoperative imaging indicating direct invasion, or intraoperative findings of direct involvement of adjacent organs by the surgeon. Patients with known metastatic disease at the time of operation were excluded from the analysis.

Clinical characteristics (age, gender, mode of presentation, symptoms, comorbid conditions, family history of CRC), preoperative workup, the operation performed, postoperative course, histological diagnoses, and follow-up were collected on a proforma.

Multivisceral resection of organs was grouped and analysed as abdominal wall, urologic (kidneys, ureter, bladder, seminal vesicles, prostate and urethra), gynaecologic (uterus, ovaries and adnexa, and vagina), hepatobiliary (liver, gallbladder, bile ducts, pancreas and duodenum, and spleen) and noncontinuous bowel (colon or small bowel).

The patient's status (whether alive or dead) was obtained from the South African Department of Home Affairs' database. Statistical analysis was performed both manually and with the Statistical Package for the Social Sciences (SPSS) v.23 software (IBM Corporation, Armonk, NY).

\section{Results}

Four hundred and ninety-four patients underwent 514 colorectal cancer operations between January 2008 and December 2013. One hundred and fifty-eight were suspected 
to have T4 disease without metastases on preoperative clinical staging or intraoperative findings. MVR was performed in 44 patients from the T4 group, in whom tumour adherence/ infiltration of another organ was observed intraoperatively. One patient had confirmed distant metastases soon after surgery and was excluded from the analysis.

While the majority of the cases were primary tumours, four were colonic recurrences, two of which were metachronous cancers (caecum and descending colon). In addition, there were two anastomotic recurrences of sigmoid cancer.

\section{Clinical characteristics of the MVR group}

These are highlighted in Table 1. The median age was 64 years (range 20-80); there was no gender difference. Twentysix patients $(59 \%)$ presented electively at the outpatient department. Intestinal obstruction and abdominal pain, occurring in $8(19 \%)$ and $5(12 \%)$ patients respectively, were the commonest presentations to the emergency department.

The most commonly reported symptoms to the outpatient department included weight loss in 26 patients $(60 \%)$, abdominal pain in $26(60 \%)$ and rectal bleeding in $18(42 \%)$. Sixty-three per cent of patients had at least one comorbid condition. Six (14\%) had a positive family history of CRC, afflicting a first-degree relative in 5 .

Preoperative staging with CT scan of the chest and abdomen was used for patients presenting electively with colon cancer, and MRI of the pelvis for those with rectal cancer (defined as tumour within $15 \mathrm{~cm}$ from the anal verge on rigid sigmoidoscopy). All but one patient who presented as emergency had preoperative CT scan. The single patient had an emergency laparotomy for an acute abdomen and generalised peritonitis.

\section{Surgery and postoperative morbidity}

Twenty-one operations were consultant-led. A consultant was present at all resections for low rectal cancer, defined as a rectal tumour situated within $10 \mathrm{~cm}$ from the anal verge. Twenty-one operations were completed by a senior registrar (SR) without a consultant colorectal surgeon present in the operating room. (In our institution, senior registrars are qualified surgeons undergoing sub-specialist colorectal surgical training). A registrar (assisted by a $\mathrm{SR})$, performed one colonic resection (2\%). Distal cancers

\begin{tabular}{lcc}
\multicolumn{2}{l}{ Table 1: Patients' clinical characteristics } & \\
\hline Characteristic & $\boldsymbol{n}=\mathbf{4 3}$ & $\mathbf{\%}$ \\
\hline Gender & 21 & 49 \\
-Male & 22 & 51 \\
-Female & & \\
Mode of presentation & 26 & 60 \\
-Elective & & \\
-Emergency & 8 & 19 \\
- Obstruction & 5 & 12 \\
- Pain & 1 & 2 \\
- Peritonitis & 3 & 7 \\
-Unknown & & \\
Symptoms & 26 & 60 \\
-Abdominal pain & 26 & 60 \\
-Weight loss & 18 & 42 \\
-Bleeding & 16 & 37 \\
-Constipation & 11 & 26 \\
-Diarrhoea & 27 & 63 \\
Comorbid illness & 6 & 14 \\
Family history &
\end{tabular}

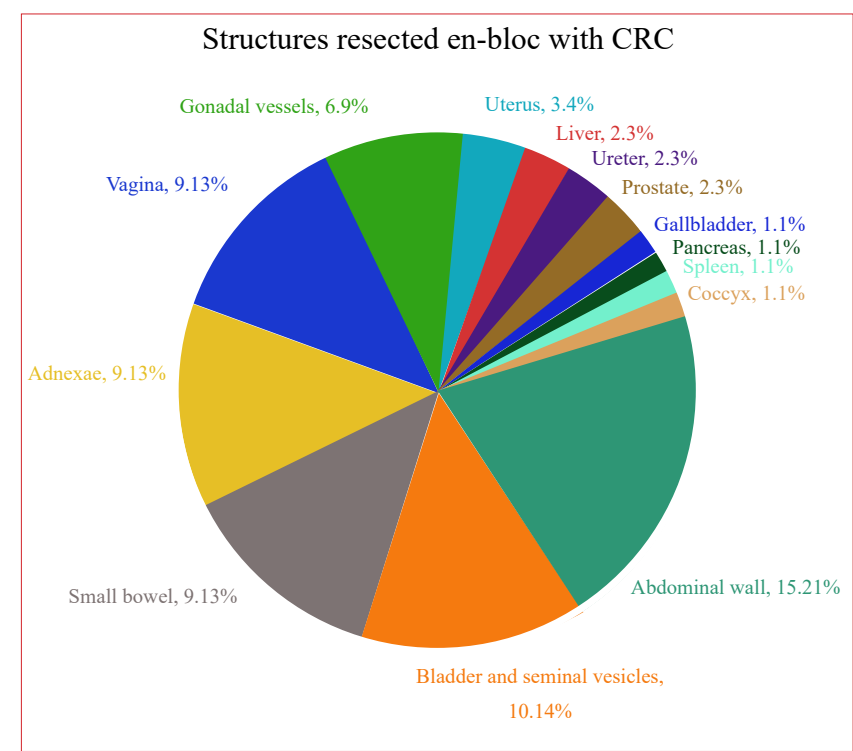

Figure 1: Structures resected en bloc with the colorectal primary

(splenic flexure and beyond) accounted for $74 \%$ of lesions. The most common extra-colonic structures resected were the abdominal wall $(21 \%)$, urinary bladder and/or seminal vesicle $(14 \%)$ and small intestine, vagina and adnexa $(13 \%)$ each (Figure 1).

Tumour spillage occurred in 11 cases $(26 \%)$. This was not significantly associated with the level of expertise of the surgeon $(p=0.78)$.

Twenty-nine patients $(67 \%)$ had a primary anastomosis following the resection of the cancer (Table 2). Primary anastomoses were made in all colonic cancer patients. Sixty per cent $(3 / 5)$ of patients with a rectosigmoid cancer and $29 \%(5 / 17)$ of those with a rectal cancer had a primary anastomosis. None of the 6 patients who received a Hartmann's procedure had their colostomy reversed.

Anastomotic leakage (defined as a peri-anastomotic gascontaining fluid collection on contrast-enhanced CT of the abdomen) occurred in $3 / 29$ patients $(10 \%)$. This was not related to the level of expertise of the surgeon $(p=0.72)$ or the site of anastomosis (intra-peritoneal vs pelvic. $p=0.61)$. Superficial site infection was the most common postoperative complication, occurring in 18 patients $(42 \%)$.

Respiratory complications were significantly higher in consultant-led cases $(p=0.04)$. Surgical site infection $(p=0.10)$, bleeding $(p=0.15)$, and anastomotic leakage $(p=0.27)$ were similar. Postoperative morbidity between the groups of multivisceral resection (abdominal wall, urologic, gynaecologic or hepatobiliary) were similar (Table 3).

\begin{tabular}{lc} 
Table 2: Post-resection reconstruction & \\
\hline Reconstruction & $\boldsymbol{n}=\mathbf{4 3}(\boldsymbol{\%})$ \\
\hline Primary anastomosis & $29(67)$ \\
Abdominoperineal resection & $8(19)$ \\
Hartmann's resection & $6(14)$
\end{tabular}

\begin{tabular}{lc} 
Table 3: Postoperative morbidity & \\
\hline Morbidity & $\boldsymbol{n = 4 3 ( \% )}$ \\
\hline Superficial wound infection & $18(42)$ \\
Respiratory tract infections & $6(14)$ \\
Anastomotic leakage' & $3 / 29(10)$ \\
Gastrointestinal bleeding & $2(5)$ \\
'Only 29 patients had an anastomosis &
\end{tabular}


There were three deaths $(7 \%)$, two from pneumonia and one from sepsis following an anastomotic leak. The median length of hospital stay (LOS) was 11 days (range 4-46 days).

\section{Neoadjuvant and adjuvant treatment}

Fifteen patients $(35 \%)$, all with rectal cancer, received neoadjuvant treatment, of which the majority had long course chemoradiation. Thirteen patients required adjuvant chemotherapy (30\%). Seven rectal cancer patients received palliative chemotherapy on account of recurrent disease or incompletely resected tumour. In this resource-constrained environment, our provincial department of health did not fund oxaliplatin during the study period. Therefore all but one of our patients were treated with 5-fluorouracil (5-FU)

\begin{tabular}{lc} 
Table 4: Histology of resection specimens & \\
\hline Histological finding & $\boldsymbol{n}=\mathbf{4 3}(\mathbf{\%})$ \\
\hline Grade & \\
Well-differentiated & $6(14)$ \\
Moderate & $25(58)$ \\
Poor & $5(12)$ \\
Mucinous & $5(12)$ \\
Complete response & $1(2)$ \\
Not stated & $1(2)$ \\
Pathological (p) T stage & \\
pT0 & $1(2)$ \\
pT1 & $1(2)$ \\
pT2 & $5(12)$ \\
pT3 & $14(33)$ \\
pT4A & $5(12)$ \\
pT4B & $16(37)$ \\
pTx & $1(2)$ \\
Pathological (p) N stage & \\
pN0 & $26(60)$ \\
pN1 & $9(21)$ \\
pN2 & $7(16)$ \\
pNx & $1(2)$ \\
Resection margin & \\
R0 & $32(73)$ \\
R2 & $9(21)$ \\
Perineural/vascular invasion & $3(7)$ \\
& $8(19)$ \\
&
\end{tabular}

and leucovorin/folinic acid (LV). The single patient received the FOLFOX regime (LV, 5-FU and oxaliplatin).

\section{Histology}

The histological characteristics of the resected specimens are summarised in Table 4.

Twenty-five tumours (58\%) were moderately-differentiated adenocarcinoma. Tumour infiltration into contiguous organs (T4b) was confirmed in only $37 \%$ of specimens. Therefore $63 \%$ of the cases where tumour involvement of adjacent organs was suspected did not have direct tumour invasion. A mean of 14 lymph nodes was retrieved per specimen (range 0-49). Twenty-six tumours (60\%) had no lymph node involvement. A complete pathological resection (R0 resection) was achieved in 32 tumours (74\%). Complete pathological response (ypT0N0) was observed in one tumour from a patient who was treated with neoadjuvant long-course chemoradiation (LCRT).

\section{Outcome and survival analysis}

After a mean length of follow-up (LOF) of 30 months (range 0-78 months), median survival was 68 months (SD 13.9). Five-year disease free (DFS) and overall survivals (OS) were $45 \%$ and $55 \%$, respectively.

Disease recurred in 17 patients (40\%): distant metastases in $16 \%$; local recurrence $14 \%$; and both $10 \%$. As at 14 January 2018, when the Department of Home Affairs database was accessed, 24 (55\%) patients were still alive, 18 (42\%) were dead and the status was not obtainable for one patient.

On multivariate analysis (Table 5), an R0 resection was a significant predictor of improved survival (Figure 2); a combination of tumour spillage, lymphovascular and perineural invasion and anastomotic leakage significantly predicted poor survival. Anastomotic leakage was also an independent predictor of local recurrence $(p=0.022)$.

Long-course chemoradiation and an $\mathrm{R} 0$ resection were significant predictors of survival in patients with a rectal primary, whereas distant recurrence indicated a poor prognosis.

Table 5: Factors affecting survival after MVR in the colon, rectum and the combined cohorts (multivariate analysis)

\begin{tabular}{|c|c|c|c|c|c|c|c|c|c|c|c|c|}
\hline \multirow{2}{*}{ Variable } & \multicolumn{4}{|c|}{ Colon } & \multicolumn{4}{|c|}{ Rectum } & \multicolumn{4}{|c|}{ Combined colon and rectum } \\
\hline & df & $p$-value & HR & CI & df & $p$-value & HR & CI & df & $p$-value & HR & CI \\
\hline $\mathrm{R} 0$ resection & 1 & 0.022 & 7.67 & $0.00-0.58$ & 1 & 0.024 & 0.04 & $0.00-0.85$ & 1 & 0.027 & 0.13 & $0.021-0.797$ \\
\hline Tumor spillage & 1 & 0.047 & 0.02 & $1.02-57.47$ & 1 & 0.602 & 0.68 & $0.16-2.93$ & 1 & 0.017 & 6.45 & $1.404-29.665$ \\
\hline $\begin{array}{l}\text { Vascular/ } \\
\text { Perineural invasion }\end{array}$ & 1 & 0.973 & 0.00 & $0.00-$ & 1 & 0.979 & 194729.31 & - & 1 & 0.012 & 22.57 & $1.880-271.024$ \\
\hline Anastomotic leak & 1 & 0.757 & 1.35 & $0.24-8.88$ & 1 & 0.253 & 4.58 & $0.34-61.98$ & 1 & 0.602 & 1.66 & $0.249-11.031$ \\
\hline Local recurrence & 1 & 0.091 & 0.17 & $0.02-1.33$ & 1 & 0.575 & 1.55 & $0.34-7.10$ & 1 & 0.697 & 1.35 & $0.585-29.903$ \\
\hline Distant recurrence & 1 & 0.052 & 7.44 & $0.99-56.12$ & 1 & 0.021 & 47.89 & $1.81-1264.24$ & 1 & 0.164 & 3.74 & $0.585-29.903$ \\
\hline Gender (F:M) & 1 & 0.062 & 5.48 & $0.92-32.76$ & 1 & 0.770 & 1.27 & $0.26-6.24$ & 1 & 0.103 & 3.07 & $0.796-11.866$ \\
\hline Adjuvant therapy & 1 & 0.509 & 0.53 & $0.08-3.52$ & - & - & - & - & 1 & 0.209 & 2.66 & $0.579-12.193$ \\
\hline $\begin{array}{l}\text { Neoadjuvant } \\
\text { therapy }\end{array}$ & - & - & - & - & - & - & - & - & 1 & 0.601 & 0.689 & $0.170-2.788$ \\
\hline Age & 1 & 0.157 & 0.96 & $0.90-1.02$ & - & - & - & - & 1 & 0.533 & 0.98 & $0.934-1.036$ \\
\hline PT & 1 & 0.359 & 0.71 & $0.33-1.49$ & - & - & - & - & - & - & - & - \\
\hline PN & 1 & 0.212 & 0.54 & $0.21-1.42$ & - & - & - & - & - & - & - & - \\
\hline LCRT & - & - & - & - & 1 & 0.047 & 0.01 & $0.00-0.94$ & - & - & - & - \\
\hline
\end{tabular}




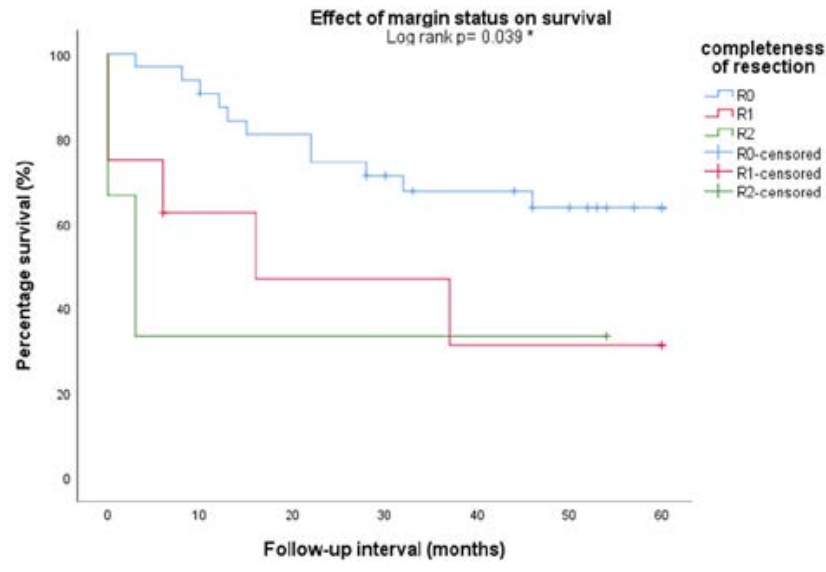

Figure 2: Kaplan-Meier curves showing the effect of pathological status of the resection margin on survival. $R 0$ - microscopically negative margin; $R 1$ - microscopically involved margin; $R 2$ - macroscopically involved margin

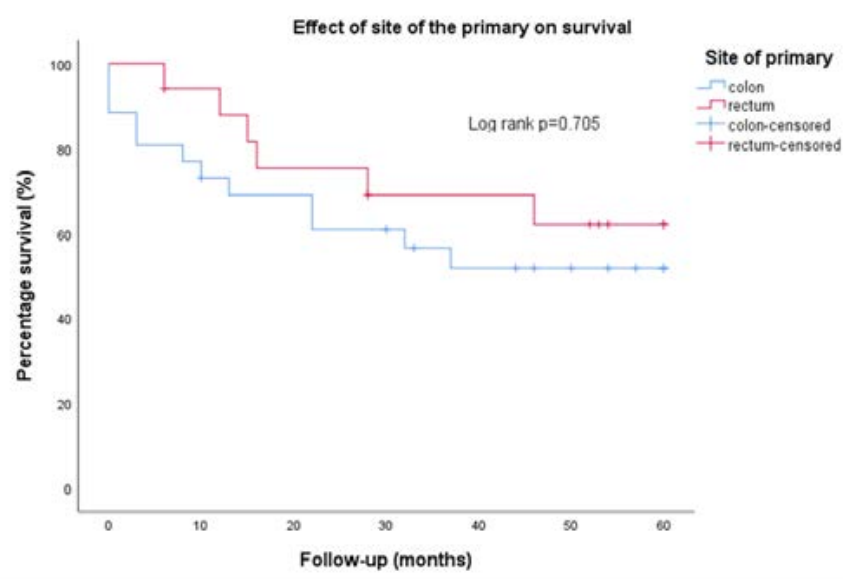

Figure 3: Kaplan-Meier curves for survival according to the site of the primary tumour

In patients with colonic cancer, an $\mathrm{R} 0$ resection was significant predictor of survival whereas tumour spillage significantly predicted a poor prognosis. There was no difference in overall survival between cancers of the colon and rectum $(p=0.705)$ (Figure 3$)$.

Seven of the 16 patients with histologically confirmed $\mathrm{T} 4 \mathrm{~b}$ had an $\mathrm{R} 1$ resection. $\mathrm{R} 1$ resection was shown to be an independent predictor of poor survival. To compare DFS and OS between patients with pT4b to those with inflammatory adhesions ( $n=27,61 \%)$, we excluded patients with an R1 resection from the comparison. Nine patients had an $\mathrm{R} 0$ resection for a true primary $\mathrm{T} 4 \mathrm{~b}(\mathrm{pT} 4 \mathrm{~b})$ tumour.

Patients with true $\mathrm{pT} 4 \mathrm{~b}$ had poorer overall survival than those with suspected T4b (mean 43 vs 46 months, $p=0.03$ ) (Figure 4). There was no difference in disease free survival $(p=0.15)$ or local recurrence $(p=0.22)$ (Figure 5).

There was no association between the level of expertise of the surgeon (consultant vs trainee) and the 5-year overall survival in either colon or high rectal resections $(p=0.11)$.

\section{Discussion}

MVR of colorectal tumours has long been considered safe and associated with equivalent survival compared with

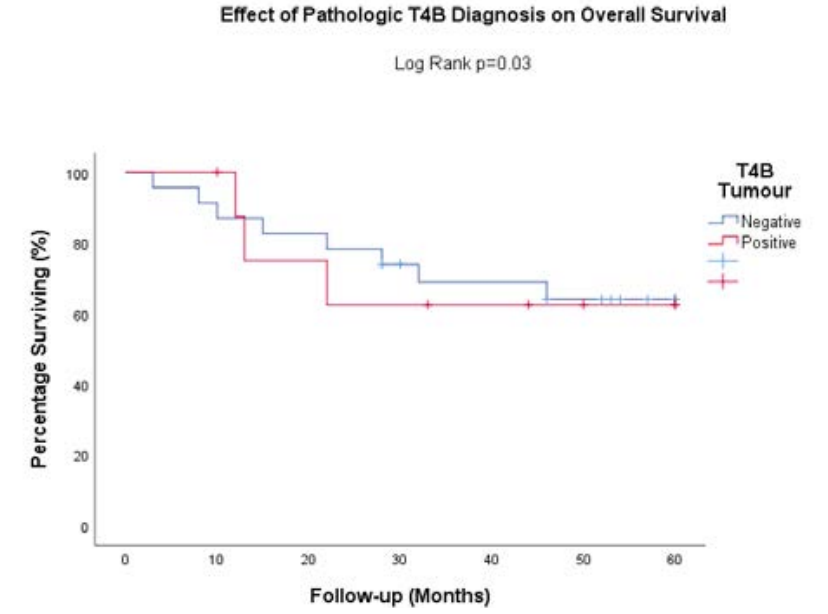

Figure 4: Kaplan-Meier curves for survival of true T4b cancer

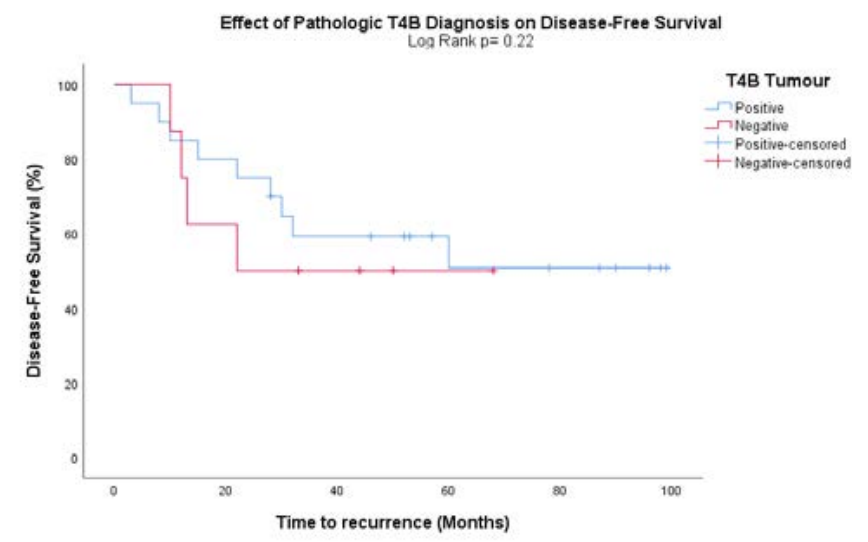

Figure 5: Kaplan-Meier curves for DFS of true T4b cancer

standard resections. ${ }^{5,7}$ Lehnert et al. demonstrated that the surgeon's level of experience was not significant in the probability of achieving cure. ${ }^{7}$ Our findings supported this and make a strong argument for teaching these complex procedures to general surgical trainees in the developing world, for whom delayed presentations are likely to form the bulk of experience.

It is important to take into consideration that in rectal cancer surgery, resection of anatomical structures beyond the mesorectal fascia poses a unique challenge. Resection of locally advanced rectal tumours including bony structures like the sacrum, or pelvic exenteration should be reserved for specialist centres with expertise and experience in these complex procedures. This study did not include any patients requiring bony resection of the pelvis or exenteration.

All patients with colorectal cancer should be managed through a multidisciplinary team (MDT). ${ }^{13}$ It is of the utmost importance that patients presenting electively with locally advanced tumours should be reviewed in an MDT. Surgical planning should make provision for any eventuality. Patients presenting in the emergency setting can often not be discussed in an MDT and the decision-making should include an experienced surgeon. All patients in this study were managed by an MDT in the preoperative and/or postoperative setting.

Only 44 patients $(28 \%)$ with preoperative T4 tumours received MVR. This was due to a number of factors, 
including presence of metastatic disease, preoperative over staging on imaging, under staging of tumours which were found to be irresectable at surgery, effective downstaging with neoadjuvant therapy, and inoperable tumours.

Neoadjuvant chemoradiation was offered to rectal cancer patients. Adjuvant chemotherapy was offered according to the standard current guidelines. ${ }^{13}$ There has been an increase in the use of neoadjuvant chemotherapy in recent years, especially after the early data of the FOxTROT trial were presented. ${ }^{14}$ Our institution did not make use of neoadjuvant chemotherapy, as it was not yet considered standard of care outside of trial setting during the study period. Oxaliplatin was not routinely available in our resource constrained healthcare setting.

Except for respiratory complications, major morbidity after procedures that were consultant- or trainee-led were similar for tumours located in the colon and high rectum. There were no differences demonstrated between the different organ groups of multivisceral resections. This finding differs from others in the literature where significant differences in complication rates and local recurrence were demonstrated..$^{15}$ The small sample size of this study may contribute to this.

Tumour spillage is a risk factor for local recurrence and a reduction in overall survival from $49 \%$ to $17 \% .^{5}$ Tumour spillage should therefore be avoided. In this series, tumour spillage occurred in 11 cases $(26 \%)$ and was a significant determinant of poor survival.

Sixteen clinical T4b specimens (37\%) were histologically confirmed pathological T4b tumours. This is in accordance with previous reports, with confirmed tumour infiltration of the resected adjacent organ in $34 \%$ to $71 \%$ of cases. ${ }^{16}$ Studies have shown that non-malignant adhesions were usually inflammatory in nature. ${ }^{7}$ Overall survival was significantly worse in patients with a true pT4b, but only by a mean of 3 months. There was no difference of DFS or local recurrence. This is in accordance with the published literature. ${ }^{17}$

Twenty-six patients $(61 \%)$ had node negative disease. This is comparable to the findings from several reports in the literature. ${ }^{18-20}$ Node negative tumours tend to have higher degrees of differentiation and/or have microsatellite instability (MSI). ${ }^{18,21}$ Whilst several studies depict lymph node involvement as an independent predictor of poor survival, it was not demonstrated to be the case in this cohort. $6,8,19,22$

A margin-negative (R0) resection is important in improving disease-free and overall survival. ${ }^{6,8,22}$ In this study, survival of patients with an R0 resection was significantly better than those with an $\mathrm{R} 1$ resection (60 months vs 16 months, $p=.039$ ).

R0 multivisceral resections are associated with higher morbidity $(33-76 \%),{ }^{7-9,11,19}$ but not with increase in 30 day mortality when compared with standard resections (0-7.7\%). ${ }^{6-8,11}$ Morbidity (51\%) and mortality (7\%) were comparable with the findings of others. Distant recurrence was an independent predictor of poor survival in rectal cancer patients, in agreement with the findings of Crawshaw et al. ${ }^{20}$

This study is limited by its retrospective nature and the small size of the cohort. However, our findings concur with the published data and demonstrate what is achievable in a resource-constrained setting.

\section{Conclusion}

MVR is feasible in a resource-constrained setting, with morbidity and mortality rates comparable to developed countries. Managing these complex patients in a multidisciplinary team should be mandatory. Outcomes appear to be independent of the level of experience, and it is recommended that surgical trainees in the developing world be exposed to these complex operations during training. A margin-negative resection should be the goal of surgery and results in improved survival.

\section{Conflict of interest}

The authors have no conflict of interest to declare.

\section{Funding source}

No funding was required.

\section{Ethical approval}

The authors declare that this submission is in accordance with the principles laid down by the Responsible Research Publication Position Statements as developed at the 2nd World Conference on Research Integrity in Singapore, 2010.

Ethics approval was obtained from the University of Cape Town Human Research Ethics Committee, reference $217 / 2015$.

\section{ORCID}

OA Oke (D) https://orcid.org/0000-0002-7362-715X

EDT Coetzee (iD https://orcid.org/0000-0001-7384-1386

C Warden (iD https://orcid.org/0000-0002-4409-6660

P Goldberg (iD https://orcid.org/0000-0003-1612-7519

A Boutall (iD https://orcid.org/0000-0002-6413-5890

\section{REFERENCES}

1. Bray F, Ren JS, Masuyer E, Ferlay J. Global estimates of cancer prevalence for 27 sites in the adult population in 2008. Int J Cancer. 2013 Mar 1;132(5):1133-45. https://doi. org/10.1002/ijc.27711.

2. Ferlay J, Soerjomataram I, Dikshit R, et al. Cancer incidence and mortality worldwide: sources, methods and major patterns in GLOBOCAN 2012. Int J Cancer. 2015 Mar 1;136(5):e35986. https://doi.org/10.1002/ijc.29210.

3. Ferlay J, Ervik M, Dikshit R, et al. GLOBOCAN 2012: Estimated Cancer Incidence, Mortality and Prevalence Worldwide in 2012 v1.0. Lyon: IARC CancerBase No11. 2012.

4. Saluja S, Alatise OI, Adewale A, et al. A comparison of colorectal cancer in Nigerian and North American patients: is the cancer biology different? Surgery. 2014 Aug;156(2):30510. https://doi.org/10.1016/j.surg.2014.03.036.

5. Gall FP, Tonak J, Altendorf A. Multivisceral resections in colorectal cancer. Dis Colon Rectum. 1987 May;30(5):33741. https://doi.org/10.1007/BF02555450.

6. Derici H, Unalp HR, Kamer E, et al. Multivisceral resections for locally advanced rectal cancer. Colorectal Dis. 2008 Jun;10(5):453-9. https://doi.org/10.1111/j.14631318.2007.01427.x.

7. Lehnert T, Methner M, Pollok A, et al. Multivisceral resection for locally advanced primary colon and rectal cancer: an analysis of prognostic factors in 201 patients. Ann Surg. 
2002 Feb;235(2):217-25. https://doi.org/10.1097/00000658200202000-00009.

8. Hoffmann M, Phillips C, Oevermann E, et al. Multivisceral and standard resections in colorectal cancer. Langenbecks Arch Surg. 2012 Jan;397(1):75-84. https://doi.org/10.1007/ s00423-011-0854-z.

9. Park S, Lee YS. Analysis of the prognostic effectiveness of a multivisceral resection for locally advanced colorectal cancer. J Korean Soc Coloproctol. 2011 Feb;27(1):21-6. https://doi. org/10.3393/jksc.2011.27.1.21.

10. Gezen C, Kement M, Altuntas YE, et al. Results after multivisceral resections of locally advanced colorectal cancers: an analysis on clinical and pathological t4 tumors. World J Surg Oncol. 2012 Feb 15;10:39-7819-10-39. https:// doi.org/10.1186/1477-7819-10-39.

11. Nakafusa Y, Tanaka T, Tanaka M, et al. Comparison of multivisceral resection and standard operation for locally advanced colorectal cancer: analysis of prognostic factors for short-term and long-term outcome. Dis Colon Rectum. 2004 Dec;47(12):2055-63. https://doi.org/10.1007/s103500040716-7.

12. Newland RC, Dent OF, Chapuis PH, Bokey EL. Clinicopathologically diagnosed residual tumor after resection for colorectal cancer. A 20-year prospective study. Cancer 1993 Sep 1;72(5):1536-42. https://doi. org/10.1002/1097-0142(19930901)72:5<1536::aidcncr2820720508>3.0.co;2-u.

13. Schmoll HJ, Van Cutsem E, Stein A, et al. ESMO Consensus Guidelines for management of patients with colon and rectal cancer. a personalized approach to clinical decision making. Ann Oncol. 2012 Oct;23(10):2479-516. https://doi:10.1093/ annonc/mds236.

14. Foxtrot Collaborative G. Feasibility of preoperative chemotherapy for locally advanced, operable colon cancer: the pilot phase of a randomised controlled trial. Lancet Oncol. 2012 Nov;13(11):1152-60. https://doi.org/10.1016/S14702045 (12)70348-0.
15. Wasmann KA, Klaver CEL, Van der Bilt JDW, et al. Subclassification of multivisceral resections for $\mathrm{T} 4 \mathrm{~b}$ colon cancer with relevance for postoperative complications and oncological risks. J Gastrointest Surg. 2019 Nov 20. https:// doi.org/10.1007/s11605-019-04426-3.

16. Nagasue $Y$, Akiyoshi T, Ueno M, et al. Laparoscopic versus open multivisceral resection for primary colorectal cancer: comparison of perioperative outcomes. J Gastrointest Surg. 2013 Jul;17(7):1299-1305. https://doi.org/10.1007/s11605013-2222-5.

17. Mohan HM, Evans MD, Larkin JO, Beynon J, Winter DC. Multivisceral resection in colorectal cancer: a systematic review. Ann Surg Oncol. 2013 Sep;20(9):2929-36. https://doi. org/10.1245/s10434-013-2967-9.

18. Eveno C, Lefevre JH, Svrcek M, et al. Oncologic results after multivisceral resection of clinical T4 tumors. Surgery 2014 Sep;156(3):669-75. https://doi.org/10.1016/j.surg.2014. 03.040.

19. Manas MJ, Espin E, Lopez-Cano M, Vallribera F, ArmengolCarrasco M. Multivisceral resection for locally advanced rectal cancer: prognostic factors influencing outcome. Scand J Surg. 2015 Sep;104(3):154-60. https://doi.org/10.1177/ 1457496914552341.

20. Crawshaw BP, Augestad KM, Keller DS, et al. Multivisceral resection for advanced rectal cancer: outcomes and experience at a single institution. Am J Surg. 2015 Mar;209(3):526-31. https://doi.org/10.1016/j.amjsurg.2014.10.014.

21. Eveno C, Nemeth J, Soliman H, et al. Association between a high number of isolated lymph nodes in T1 to T4 N0M0 colorectal cancer and the microsatellite instability phenotype. Arch Surg. 2010 Jan;145(1):12-7. https://doi.org/10.1001/ archsurg.2009.224.

22. Croner RS, Merkel S, Papadopoulos T, et al. Multivisceral resection for colon carcinoma. Dis Colon Rectum. 2009 Aug;52(8):1381-6. https://doi.org/10.1007DCR.0b013e3181a b580b. 